\title{
Cure Advancement of Urethane Networks Using a Sigmoidal Chemorheological Model
}

\author{
F. Teyssandier, ${ }^{1}$ B.J. Love ${ }^{1,2,3}$ \\ ${ }^{1}$ Department of Materials Science and Engineering, University of Michigan, Ann Arbor, Michigan 48109 \\ ${ }^{2}$ Department of Biomedical Engineering, University of Michigan, Ann Arbor, Michigan 48109 \\ ${ }^{3}$ Department of Biologic and Materials Sciences (Dentistry), University of Michigan, Ann Arbor, \\ Michigan 48109
}

\begin{abstract}
Archived measurements of urethane rheology undergoing crosslinking at 45,55 , and $65^{\circ} \mathrm{C}$ were reanalyzed by a sigmoidal mathematical model. The phenomenological sigmoidal model, with two kinetic parameters associated with cure advancement coupled with parameters for the initial viscosity and the terminal viscosity of the network, captures the nonlinearities in dynamic viscosity during polymerization and were similarly compared with a power law model. The interpretation of the gel time was determined using both models, with the sigmoidal model interpretation of the gel time being defined in terms of the other kinetic constants. The takeaway message is that there is very little difference in the quality of the fit between the power law model and the sigmoidal model and the experimental dynamic viscosity data. One would need a more comprehensive dataset to make a larger comparison as to which model actually represents viscosity more accurately. POLYM. ENG. SCI., 50:499-503, 2010. ( 2009 Society of Plastics Engineers
\end{abstract}

\section{INTRODUCTION}

Rheological changes arising during network polymerization, linear polymerization, and the transition from small molecule behavior to a polymeric form have been studied by both phenomenological and predictive molecular models. These systems have been characterized by dynamic rheological evaluations with both chain length and the rate of lengthening regulating the continued progression to long chain behavior. Fundamental relationships based on scaling concepts and more empirical $\eta$-log molecular weight relationships have the strongest molecular basis for how viscosity changes during polymerization

Correspondence to: Brian J. Love; e-mail: bjlove@umich.edu DOI 10.1002/pen.21560

Published online in Wiley InterScience (www.interscience.wiley.com) (C) 2009 Society of Plastics Engineers
$[1,2]$. Dunston [3] and later Flory [4] had originally suggested that the weight average molecular weight most accurately represents the state of the expanding polymer and its incipient viscosity, as indicated in Eq. 1 .

$$
\log \eta=A+C M_{w}{ }^{1 / 2}
$$

This relationship is similar to that found in longer, linear polymers, which show a power law relationship with molecular weight with a power law exponent of $\sim 3.4$ [5].

Strictly kinetic models of polymerization have focused on dynamic viscosity and related it to various experimental conditions using the power law model [6-10], and other empirical models for advancement [11]. Halley and Mackay published a comprehensive list of rheological advancement models, ranging from molecular models to other models based on kinetics, thermodynamics relating to activation energies for advancement and other strictly empirical models as well [11]. Regimes of conversion are attributed to chemical reaction kinetics in the dilute monomer state, and again approaching the gel point and beyond, where the physics of conversion are quite different [11]. They have shown specific resin systems that have been evaluated with each model. Although it is possible that each model is generally more applicable, it's worth noting that specific models may be restricted to describe the physics of one type of polymerizing resin.

We've focused on an alternative model that replicates the features observed in most rheological advancement curves. One feature is a sigmoidal asymptotic limit in viscosity, which could either be an artifact of torque limitations in the rheometer or it could be a threshold of viscosity at high conversion, when further reaction is more sluggish. The probing of this model includes much of our own work focused primarily on both radical polymerized acrylates [12,13] and epoxy resins [14-16]. The power law model does a fine job of characterizing the time 
dependent viscosity [10]. With refinements, we have investigated the use of nonlinear models that describe the transition regions during the initial conversion stage and again at higher conversion where the chain length is a drag on the rate of polymerization as monomer content is extinguished. We have found the modified Boltzmann sigmoidal model, shown in Eq. 2, represents viscosity advancement extremely well both for photopolymerized acrylates [12], thermally advanced acrylates [17], and also for epoxy resins formulated as chip under fill materials $[14,15]$.

$$
\log \eta(t)=\log \eta_{\infty}+\frac{\log \left(\eta_{0}\right)-\log \left(\eta_{\infty}\right)}{\left(1+e^{\left(\frac{t-t_{0}}{\Delta t}\right)}\right)}
$$

where $\eta_{0}$ and $\eta_{\infty}$ represents forms of an initial and some type of terminal viscosity more related to the torque limit of the rheometer, $t_{0}$ is an induction time for viscosity to achieve $50 \%$ conversion on a log scale, and $\Delta t$ is the time constant inversely proportional to the slope of the curve at $50 \%$ conversion. Both the molecular and phenomenological approaches have merit.

Every model also seems to have its drawbacks. The molecular based models are based on molecular weight or chain length, yet there are few studies, which have attempted to characterize in situ molecular weight during conversion in any sort of functional way. Lipshitz and Macosko performed several evaluations based on molecular weight that have shed light on the relative activation energies required to drive further conversion [18, 19], which increase with conversion. These studies follow other phenomenological correlations describing the melt viscosity and its power law relationship with increasing conversion above the gel point. The kinetic models can yield a dynamic viscosity, which can be used effectively, but subtle changes in processing or formulation conditions can quickly erode the confidence in the parametric models.

Practically, the determination of which models (molecular vs kinetic) are the most predictive for thermoset processing procedures like reactive injection molding, reactive extrusion, thermoforming, and resin infiltration suggest that something can be learned by mining prior to chemorheology results published in the literature as a comparison. We evaluated how the sigmoidal model stacked up against other prior work modeling resin rheological advancement. Data were available from Lipshitz and Macosko on the network formation of polyurethanes [19]. We compared the original analysis [19] with a Boltzmann sigmoidal model. This dataset was appealing because the raw dynamic viscosity data was published in tabular form and adequate comparisons could be made.

The primary analytical focus of Lipshitz and Macosko was to determine the activation energies for flow which also regulate the speed at which reactive endgroups encountered one another [19]. This particular study was interesting in that they also determined the instantaneous molecular weight and $\eta(t)$ during polymerization. They polymerized these mixtures at various temperatures and determined effective activation energies both for viscous flow and for chemical reaction based on how the kinetics of conversion were affected by temperature. The activation energy for polymerization was $40 \mathrm{~kJ} / \mathrm{mole}$ initially but rose to $\sim 120 \mathrm{~kJ} / \mathrm{mole}$ at higher conversion [19]. We chose to evaluate these dynamic viscosity datasets in terms of both a power law model and the sigmoidal model.

\section{EXPERIMENTAL}

The original published experiments used an e-caprolactone based trial (Union Carbide PCP 0300) with 99\% primary $\mathrm{OH}$ groups [19]. The urethane linkages were formed using 1-6 hexamethylene diisocyanate reacted in the presence of a nonstoichiometric quantity of tripropylene glycol to lower the NCO concentration to $18.55 \%$. The following references provide more detail [18, 19].

Rheology was characterized by a Rheometrics Mechanical Spectrometer using a 50-mm diameter cone and plate assembly. The components were mixed offline and within $3 \mathrm{~min}$, an appropriate sample was transferred to the rheometer after which measurement commenced. Experiments were run at 45,55 , and $65^{\circ} \mathrm{C}$, and were compared with other analyses probing $\mathrm{MW}$ changes and the overall kinetics of reaction with temperature. It was identified separately that there was little shear thinning occurring at least up to $10^{4} \mathrm{~Pa} \cdot \mathrm{s}$.

The results in Ref. 19 were inputted into Microcal ORIGIN (Origin Lab; Northampton, MA, USA), which has a sigmoidal plot and a normal power law analysis. Power law exponents were determined in the two regimes of MW advancement, and sigmoidal plot parameters fixing up to two of the four variables in the analysis.

\section{RESULTS AND DISCUSSION}

Lipshitz and Macosko, in their original article, showed a power law plot but did not determine power law exponents for the different polymerization temperatures [19]. We determined these and compared these models with the modified Boltzmann sigmoidal analytical model.

Conceptually, the power law analysis is a curve fitting exercise to match up two distinct zones of cure advancement as in Fig. 1. The junction of the interface between model 1 at lower conversion and model 2 at higher conversion is identified as the gel point. The power coefficients for both high and low conversion are shown in Table 1. The low conversion regime at all polymerization temperatures is generally attributed to the stable polymerization region based on the power law exponent analysis by Cioffi et al. where the exponent ranges from $\sim 5$ to 7 $[7,8]$. Progressing past the gel point, the high conversion region is interpreted as Trommsdorff Region, where the power law exponent is $\gg 7$ [8]. 


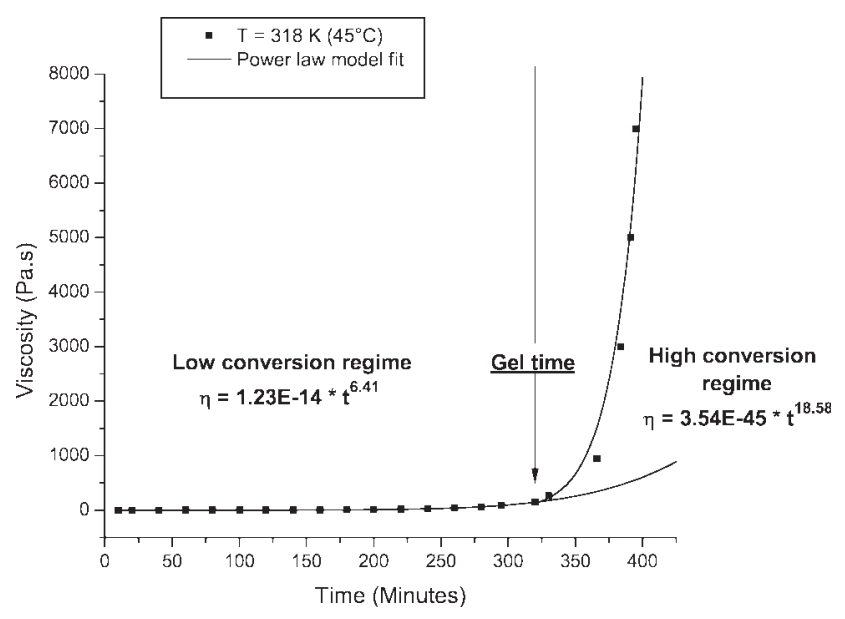

FIG. 1. Plot of viscosity vs time at $318 \mathrm{~K}$ for the polyurethane data listed in Ref. 19 with the corresponding power law fit.

Figure 2 shows different curves for the power law model using rectilinear coordinates for the three different cure temperatures, with the corresponding power law matches for $t_{\mathrm{gel}}$. These fits are compared with a modified sigmoidal model that took the form of $E q .3$ as shown below. Equation 2 tended to yield asymptotic limits for $\eta_{\infty}$ that were unrealistic.

$$
\eta(t)=\eta_{\infty}+\frac{\eta_{0}-\eta_{\infty}}{\left(1+e^{\left(\frac{t-t_{0}}{\Delta t}\right)}\right)}
$$

The initial viscosity, $\eta_{0}$, requires an activation energyrelated reduction in the extrapolated initial viscosity at time zero with increased temperature that was originally identified by Lipshitz and Macosko [19]. The temperature sensitivity of $\eta_{0}$ also needs to be included, as shown in Eq. 4.

$$
\eta(t)=\eta_{\infty}+\frac{A e^{-E a / R T}-\eta_{\infty}}{\left(1+e^{\left(\frac{t-t_{0}}{\Delta t}\right)}\right)}
$$

Using Lipshitz's first measurements as the viscosity at time 0 , the activation energy for the viscosity reduction

TABLE 1. Parameters associated with the power law model for

\begin{tabular}{|c|c|c|c|c|c|c|}
\hline & \multicolumn{6}{|c|}{ Temperature $(\mathrm{K})$} \\
\hline & \multicolumn{2}{|l|}{318} & \multicolumn{2}{|c|}{328} & \multicolumn{2}{|l|}{338} \\
\hline & Exponent & $R^{2}$ & Exponent & $R^{2}$ & Exponent & $R^{2}$ \\
\hline $\begin{array}{l}\text { Low conversion } \\
\text { regime }\end{array}$ & 6.41 & 0.994 & 7.86 & 0.993 & 9.34 & 0.983 \\
\hline $\begin{array}{l}\text { High conversion } \\
\text { regime }\end{array}$ & 18.58 & 0.955 & 17.56 & 0.973 & 30.89 & 0.972 \\
\hline Gel time $(\min )$ & \multicolumn{2}{|l|}{320.4} & \multicolumn{2}{|l|}{196} & \multicolumn{2}{|c|}{114.7} \\
\hline $\begin{array}{c}\text { Viscosity at gel } \\
\text { point }(\mathrm{Pa} \cdot \mathrm{s})\end{array}$ & \multicolumn{2}{|l|}{148} & \multicolumn{2}{|l|}{230} & \multicolumn{2}{|l|}{250} \\
\hline
\end{tabular}
gelation as a function of temperature.

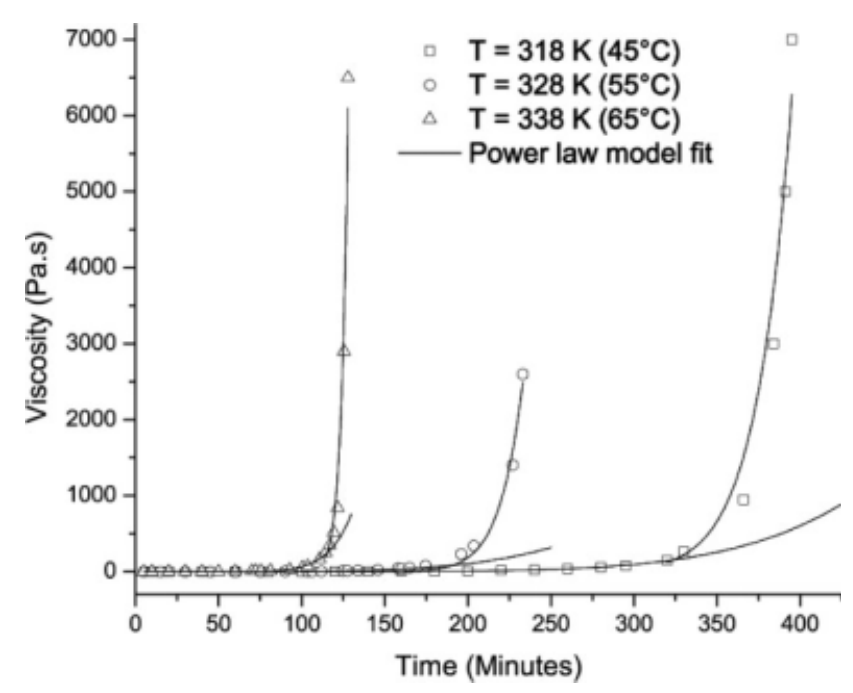

FIG. 2. Plot of viscosity vs time at 318,318 , and $338 \mathrm{~K}$ showing the junction of pregel and postgel power law fits.

found to be $39.2 \mathrm{~kJ} / \mathrm{mole}$, comparable with that identified by Lipshitz as $41.65 \mathrm{~kJ} / \mathrm{mole}$ [19]. The initial front factor, A, was $1.16 \times 10^{7} \mathrm{~Pa} \cdot \mathrm{s}$, again comparable to Lipshitz who found $4.75 \times 10^{8} \mathrm{~Pa} \cdot \mathrm{s}$ [19]. The datasets do not show a dip in viscosity during network polymerization, so either the reaction is athermal or the rate of polymerization is sufficiently low that its exotherm is less of a factor on the local temperature. Any dip in viscosity is unaccounted for in the sigmoidal model.

The value for, $\eta_{\infty}$ is an extrapolation which we assigned as $1 \times 10^{5} \mathrm{~Pa} \cdot \mathrm{s}$, similar to other network structures we had analyzed earlier [17]. The physical significance of $\eta_{\infty}$ is an open question, particularly because commonly accepted theories of gelation point to an infinite viscosity, whereas experimental measurements are limited particularly at high conversion due to the torque limit of each rheometer. We fixed the perceived terminal viscosity, $\eta_{\infty}$, and kept the time constants as variables. Separately, sensitivity analyses fixed $\eta_{\infty}$ with values as high as 100 times higher than $1 \mathrm{E} 5 \mathrm{~Pa} \cdot \mathrm{s}$, tracking both the time constants and the shape of the curve. Functionally, the slope in the gel cure region is related to $\Delta \eta / \Delta t$. Figure 3 shows the fits for the modified sigmoidal model based on $E q$. 4. It is conjecture to argue whether the power law or sigmoidal model is better at representing this dataset without performing more measurements at higher viscosities. What is clear from the analysis is that the sigmoidal model is not very sensitive to the extrapolated endpoint, $\eta_{\infty}$. Little difference in correlation coefficient was observed by these perturbations in the selection of the terminal viscosity, and while the time constants were extended with higher terminal viscosity, the overall shape differences in the transient viscosity curves were unimpressive.

The kinetic time constants associated with sigmoidal analysis assigning $\eta_{\infty}$ at $1 \times 10^{5} \mathrm{~Pa} \cdot \mathrm{s}$ are shown in Table 2 . The time constants are both shorter as the curing temperature 


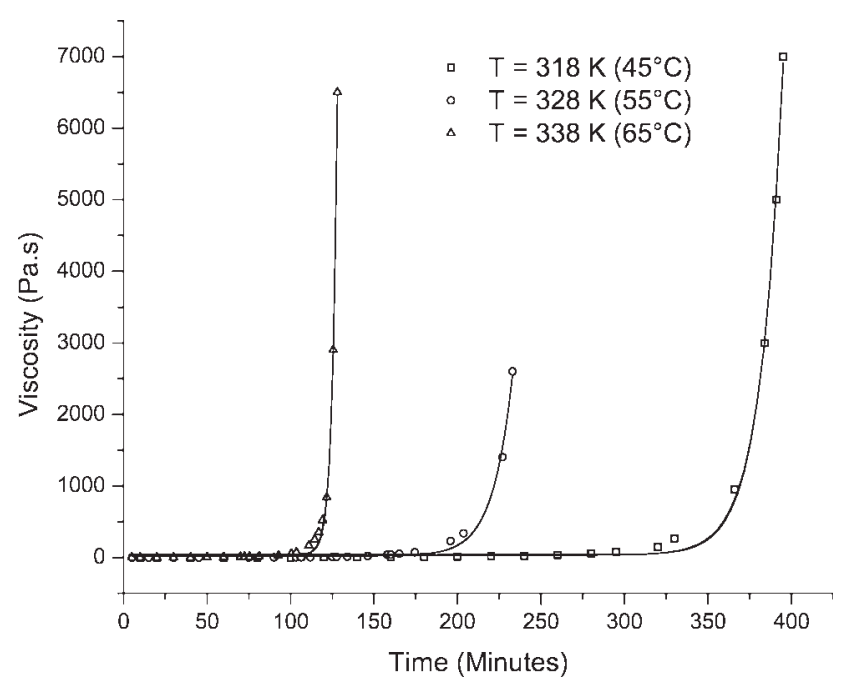

FIG. 3. Plot of viscosity vs time shown in Figure 2 with the sigmoidal analyses with the parameters identified in Table 2 .

increases correlating with the increased reactivity. Comparing these to a simple power law model in Table 1 also yields a time constants at each temperature associated with the demarcation between small molecule and larger molecule behavior where diffusion limitations are observed.

The sigmoidal model does not accommodate a distinct time point in the dynamic viscosity attributed to the normal gel time determined by the power law. Theoretically, the gel time is related to the induction time required nominally to attain $70 \%$ conversion [20]. What's arguably more useful from a processing perspective is an "engineering gel time" in terms of $t_{0}$ and $\Delta t$ from the sigmoidal analysis. The engineering gel time, $t_{\mathrm{gel}}$, is interpreted as the intersection of the extrapolated dynamic viscosity slope rise to a threshold viscosity arbitrarily assigned as $100 \mathrm{~Pa} \cdot \mathrm{s}$, shown in $E q .5$,

$$
t_{\mathrm{gel}}=t_{0}-x * \Delta t
$$

where $x$ is a scalar relating to the length of the $\Delta t$ interval needed to intersect a fixed viscosity arbitrarily assigned here as a threshold viscosity of $100 \mathrm{~Pa} \cdot \mathrm{s}$. This threshold viscosity is high enough to demarcate the gel region from the smaller molecule region using the sigmoidal curve

TABLE 2. Parameters associated with the sigmoidal model as a function of temperature including the identified gel time.

\begin{tabular}{lccc}
\hline & \multicolumn{3}{c}{ Temperature $(\mathrm{K})$} \\
\cline { 2 - 4 } & 318 & 328 & 338 \\
\hline$R^{2}$ & 0.998 & 0.992 & 0.998 \\
$\eta_{0}(\mathrm{~Pa} \cdot \mathrm{s})$ & 0.32 & 0.207 & 0.133 \\
$\eta_{\infty}(\mathrm{Pa} \cdot \mathrm{s})$ & $1 . \mathrm{E}+05$ & $1 . \mathrm{E}+05$ & $1 . \mathrm{E}+05$ \\
$t_{0}(\mathrm{~min})$ & 428.65 & 277.11 & 136.06 \\
$\Delta t(\mathrm{~min})$ & 12.92 & 12.07 & 3.01 \\
$t_{\mathrm{gel}}(\mathrm{min})$ & 367 & 220 & 122 \\
\hline
\end{tabular}

TABLE 3. Scalar parameter, $x$, as a function of temperature linking the sigmoidal model to an identified gel time.

\begin{tabular}{lccr}
\hline & \multicolumn{3}{c}{ Temperature (K) } \\
\cline { 2 - 4 } & 318 & 328 & 338 \\
\hline$X$ & 4.70 & 4.69 & 4.34 \\
\hline
\end{tabular}

and allow for objective interpretations for the time constants. The value of scalar, $x$, in Eq. 5, is shown in Table 3 for the data published by Lipshitz and Macosko [18, 19]. The scalar was invariant with the cure temperatures evaluated. The similar curve shape and the same interpreted value of $x$ with temperature suggest that the polymerization mechanism is the same. An alternative regime of polymerization might trigger a different scalar. Comparing the sigmoidal model with the power law model, both require some sort of interpretation in identifying $t_{\text {gel }}$.

The scarcity of higher viscosity measurements raises the potential that the $\eta_{\infty}$ asymptote needs refinement. It's interesting to note that in earlier studies, logarithmic sigmoidal viscosity advancement models were more accurate for radical polymerization mechanisms using acrylates $[12,17]$ as well as for epoxy resins $[12,15]$. The step polymerization from the monomer for the polyurethanes by Lipshitz and Macosko shows that, perhaps, the log sigmoidal is not as generic as a model for viscosity advancement. Lipshitz and Macosko clearly show that with advancement, there the barrier for continued reaction rises as evidenced by the higher interpreted activation energy for flow [19]. Perhaps, radical polymerizing resins donot have the same activation energy dependence on flow.

\section{CONCLUSIONS}

Published in situ viscosity advancement data of polyurethane network polymerization at three temperatures were reanalyzed using a sigmoidal model, and the results were compared with a power law model for interpreting the gel point. Power law coefficients of 5-10 were determined during stable polymerization transitioning to above 17 in the gel at higher conversion. Our determination of kinetic time constants, as a function of temperature using a modified sigmoidal model fixing $\eta_{0}$ and $\eta_{\infty}$, allowed the determination of an algorithm to identify an engineering gel point. The gel times are similar between the power law and sigmoidal models, with both models requiring some sort of interpretation. The power law model requires an interpretation of the junction region, and the sigmoidal model requires a threshold viscosity above which the gel is growing in structure and strength.

\section{REFERENCES}

1. M. Kamal and S. Sourour, Polym. Eng. Sci., 13, 59 (1973).

2. M.R. Kamal, Polym. Eng. Sci., 14, 231 (1974). 
3. A.E. Dunston, Z. Phys. Chem., 56, 370 (1906).

4. P.J. Flory, J. Am. Ceram. Soc., 62, 1057 (1940).

5. F.G. Mussatti and C.W. Macosko, Polym. Eng. Sci., 13, 236 (1973).

6. M. Cioffi, K.J. Ganzeveld, A.C. Hoffmann, and L. Janssen, Polym. Eng. Sci., 44, 179 (2004).

7. M. Cioffi, K.J. Ganzeveld, A.C. Hoffmann, and L. Janssen, Polym. Eng. Sci., 42, 2383 (2002).

8. M. Cioffi, A.C. Hoffmann, and L. Janssen, Polym. Eng. Sci., 41, 595 (2001).

9. A.Y. Malkin and S.G. Kulichikhin, Adv. Polym. Sci., 101, 217 (1991).

10. B.J. Love and F. Piguet-Ruinet, J. Appl. Polym. Sci., 106, 3605 (2007).

11. P.J. Halley and M.E. Mackay, Polym. Eng. Sci., 36, 593 (1996).

12. B.J. Love, F. Piguet-Ruinet, and F. Teyssandier, J. Polym. Sci. Part B: Polym. Phys., 46, 2319 (2008).
13. F. Piguet-Ruinet and B.J. Love, J. Appl. Polym. Sci., 107, 1523 (2008).

14. B.J. Love, F. Teyssandier, Y.Y. Sun, and C.P. Wong, Macromol. Mater. Eng., 293, 832 (2008).

15. F. Teyssandier, Y.Y. Sun, C.P. Wong, and B.J. Love, Macromol. Mater. Eng., 293, 828 (2008).

16. F. Teyssandier, M. Ivankovic, and B.J. Love, J. Appl. Polym. Sci. (2009) in press.

17. F. Teyssandier and B.J. Love, MMA Polymerization and its Influence on in Situ Resin Viscosity Using Other Mathematical Models of Chemorheology, Unpublished (2008).

18. S.D. Lipshitz and C.W. Macosko, J. Appl. Polym. Sci., 21, 2029 (1977).

19. S.D. Lipshitz and C.W. Macosko, Polym. Eng. Sci., 16, 803 (1976).

20. C.W. Macosko and D.R. Miller, Macromolecules, 9, 199 (1976). 